\title{
Molecular characterization of high performance inbred lines of Brazilian common beans
}

\author{
P.C.B. Cardoso ${ }^{1,2}$, M.M. Veiga ${ }^{1,2}$, I.P.P. de Menezes $^{1,3}$, P.A.M.R. Valdisser ${ }^{1}$, \\ T.C.O. Borba ${ }^{1}$, L.C. Melo ${ }^{1}$, M.J. Del Peloso ${ }^{1}$, C. Brondani ${ }^{1}$ and \\ R.P. Vianello ${ }^{1}$ \\ ${ }^{1}$ Laboratório de Biotecnologia, Embrapa Arroz e Feijão, \\ Santo Antônio de Goiás, GO, Brasil \\ ${ }^{2}$ Instituto de Ciências Biológicas, Universidade Federal de Goiás, \\ Goiânia, GO, Brasil \\ ${ }^{3}$ Laboratório das Ciências Biológicas, Instituto Federal Goiano, \\ Urutaí, GO, Brasil \\ Corresponding author: R.P. Vianello \\ E-mail: rosana.vianello@embrapa.br
}

Genet. Mol. Res. 12 (4): 5467-5484 (2013)

Received May 22, 2012

Accepted September 27, 2012

Published February 6, 2013

DOI http://dx.doi.org/10.4238/2013.February.6.4

\begin{abstract}
The identification of germplasm genetic variability in breeding programs of the common bean (Phaseolus vulgaris) is essential for determining the potential of each combination of parent plants to obtain superior genotypes. The present study aimed to estimate the extent of genetic diversity in 172 lines and cultivars of the common bean by integrating five tests of value for cultivation and use (VCU) that were conducted over the last eight years by the breeding program of Embrapa Arroz e Feijão in Brazil. Nine multilocus genotyping systems composed of 36 fluorescent microsatellite markers distributed across 11 different chromosomes of the common bean were used, of which 24 were polymorphic. One hundred and eighty-seven alleles were identified, with an average of 7.79 alleles per locus and an average gene diversity of 0.65 . The combined probability of identity for all loci was $1.32 \times 10^{-16}$. Lines that are more genetically divergent
\end{abstract}


between the selection cycles were identified, allowing the breeding program to develop a crossbreed between elite genotypes with a low degree of genetic relatedness. $H_{\mathrm{E}}$ values (ranging from 0.31 to 0.63 ) and the genetic differentiation among the VCU tests $\left(F_{\mathrm{ST}}\right.$ of 0.159$)$ supports for new strategies to increase the genetic base from which the program is conducted. Private alleles $(26 \%)$ were identified and can be directly incorporated into the gene pool of cultivated germplasm, thereby contributing effectively to the expansion of genetic diversity in this bean-breeding program.

Key words: Phaseolus vulgaris; Breeding; Genetic diversity; SSR markers

\section{INTRODUCTION}

The common bean (Phaseolus vulgaris), that belongs to the family Leguminosae, subfamily Papilionoideae, tribe Phaseoleae, and genus Phaseolus is considered the most important grain legume for human consumption in the world (Broughton et al., 2003). The common bean is cultivated in 113 countries and has an estimated worldwide production of 19.51 million tons. The common bean is grown annually on an average of 27 million hectares (ha) (FAO, 2008), and represents $37 \%$ of all legumes consumed in the world. Sixty percent of the world's production of the common bean is concentrated in six countries: Brazil, India, China, Myanmar, Mexico, and the United States. Currently, Brazil is the world's largest producer of this legume, representing approximately $20 \%$ of global production and occupying the second largest area of cultivation (4.01 million ha) behind India ( 8.60 million ha). Brazil's production during the 2010/2011 season yielded a total of 3.51 million tons of beans. The yield for the $2020 / 2021$ season is expected to reach 3.82 million tons ( $0.9 \%$ increase), which is linked to a $0.83 \%$ projected reduction in planting area and increased productivity (MAPA, 2011). However, both projections for production and consumption in Brazil for the period between 2011 and 2021 suggest a demand for imports between 150,000 and 200,000 tons (CONAB, 2008). Beans are considered an excellent source of protein and provide iron, phosphorus, magnesium, manganese, and, to a lesser extent, zinc, copper, calcium, and B vitamins. In addition, beans have high amounts of fiber and complex carbohydrates (Broughton et al., 2003).

The common bean is cultivated in almost all Brazilian states in various soil and climatic conditions and in different seasons and crop systems. Beans are grown in both subsistence crops, which account for $70 \%$ of the country's production, and in highly technical crops (Borém and Carneiro, 2006). Among the producing states, Paraná has the highest production (20\%), followed by the States of Minas Gerais (15\%), Bahia (10\%), São Paulo (10\%), Goiás (8\%), Santa Catarina (7\%), and Rio Grande do Sul (5\%), which together represent $75 \%$ of the national production (Ferreira et al., 2006). A regional preference for grain type is found among the Brazilian population. For example, black beans are consumed mainly in the State of Minas Gerais, and the demand for the carioca beans, currently considered the most highly consumed bean in Brazil, is extremely high in the States of Rio de Janeiro, Santa Catarina, and Rio Grande do Sul. Thus, common bean breeding programs have focused on the development and indication of new and improved lines due to the constant demand for increasingly productive cultivars with better grain quality, resistance to major diseases and adaptability to local climatic conditions and soil heterogeneity. The cultivation of specialty grains of beans, 
such as alubia, cranberry, dark red kidney, and pinto, as alternative products with higher added value in the Brazilian market has increased. However, the cultivation of specialty grains is still considered sparse and incipient when compared to the demand for the carioca grain (Bueno et al., 2011). Brazil exported 17,000 tons of beans in 2011, a $400 \%$ increase over the previous year (CONAB, 2008). This increase demonstrates a growing demand for business opportunities for this crop in the international market. Thus, common bean breeding programs should also be synchronized with the supply chain by investing in the research and development of varieties with export potential.

The development of new cultivars through breeding programs is considered a long process with a high cost due to the many steps involved during the course of successive selections and evaluations. A developed cultivar must be distinguished from other existing cultivars by easily identifiable characteristics, and these traits may include morphological, physiological, biochemical, or other characteristics considered sufficient for cultivar identification (MAPA, 2010). In addition, the genetic material must be registered and protected following the instructions provided by Plant Variety Protection Law No. 9456, which has been regulated in Brazil since 1997 to ensure that the breeders will have the intellectual property rights to the cultivars and their derivatives. Many requirements are necessary to register a cultivar, such as testing for distinctness, uniformity, and stability. In addition, breeders should perform value for cultivation and use (VCU) testing to characterize the lines. This test corresponds to the last stage of the breeding program of a new cultivar. Several agronomic traits, such as the cultivar's properties for use in agricultural, industrial, commercial, and consumer activities, are used to evaluate the performance of the lines before its release. The VCU test is conducted according to the National Register of Cultivars/Ministry of Agriculture, Livestock and Supply norms. After the test is performed, the lines that display higher performances than the reference cultivars included in the VCU test are identified. Currently, the public sector is responsible for more than $60 \%$ of the registered cultivars in Brazil (Marinho et al., 2011).

The genetic characterization of common bean lines that satisfy the VCU tests has not been reported in the literature. Thus, the use of molecular tools can help breeders to accurately evaluate the level of genetic variability found in the tests and to identify genetic material that may be used for the registration and protection of cultivars. Microsatellite markers have characteristics that bring advantages over other genetic markers, as described by several authors (Gupta et al., 1996). Recently, microsatellite markers have been used for genotyping in systems whereby the simultaneous amplification of several loci is possible using a semiautomated detection system. The development of these systems is not a trivial procedure and requires a detailed prior investigation of the loci that will encompass the genotyping panels. This procedure is performed to adopt criteria for marker selection, such as determining the specificity and size of the amplified products, estimates of locus content, and forensic parameters including the probability of identity, power exclusion, and reduced complementarity between the primers combined in a single panel.

Microsatellite markers are currently the main common markers used to estimate genetic profiles and constitute the international standard for forensic genetic research (Butler, 2006). Automated genotyping systems using microsatellites are available for genetic analyses in many plant species, such as rice (Pessoa-Filho et al., 2007), soybean (Sayama et al., 2011), and the common bean (Masi et al., 2003). These systems have several advantages over conventional genotyping methods that are based on polyacrylamide gels. For example, simultaneous analyses can be performed on a greater number of microsatellite loci, the time 
required to genotype a large number of subjects can be reduced, the data collected may be more accurate due to the addition of an internal marker in each sample, and a greater agility and security can be achieved during the data processing.

The present study aimed to establish multiplex genotyping operational systems based on microsatellite markers with higher informative power, followed by the molecular characterization of the genetic variability of common bean lines that were part of five VCU tests performed by Embrapa Arroz e Feijão breeding program between 2003 and 2012.

\section{MATERIAL AND METHODS}

\section{Plant material}

A total of 172 common bean accessions in bulks of five plants were analyzed, including the lines under evaluation (150) and control cultivars (22) that were part of the five tests for the VCU conducted in the 2003/2004, 2005/2006, 2007/2008, 2009/2010, and 2011/2012 crop years at Embrapa Arroz e Feijao. The 2003/2004 VCU test comprised 62 accessions (54 lines and 8 controls). The 2005/2006 VCU test comprised 37 accessions, of which 25 belonged to the line groups, and the remaing 12 were controls. The 2007/2008, 2009/2010, and 2011/2012 VCU tests comprised 23 (13 carioca and 10 black), 23 (13 carioca and 10 black) and 25 accessions (15 carioca and 10 black), respectively, in addition to the controls analyzed in each group. A detailed description of each VCU test and the controls is displayed in Table 1.

\section{Plant material collection and extraction of genomic DNA}

Samples of young trifoliate leaves from each of the common bean accessions evaluated in VCU tests were collected 20 days after planting in the experimental field. The procedure was performed in five steps in accordance with the year and planting period of the test. DNA extraction and quantification were performed as described by Ferreira and Grattapaglia (1998), with some modifications. Plant tissue maceration was performed in $1.5-\mathrm{mL}$ tubes with a porcelain bead using a FastPrep instrument (model FP120 Thermo Electron Corporation). After extraction, the DNA was diluted to final concentration of $3 \mathrm{ng} / \mu \mathrm{L}$.

\section{Microsatellite genotyping}

Nine multilocus genotyping systems based on 36 microsatellite markers labeled with the fluorescent dyes HEX, 6-FAM, and NED were used in the experiments. The selection of markers for co-amplification systems and analysis of forensic performance was performed according to the procedure described by Cardoso (2008). Of the 36 markers used (Table 2), 21 belonged to the BM series (Gaitán-Solís et al., 2002) and 15 belonged to the PvBr series (Buso et al., 2006). The microsatellite amplification reactions were performed using the QIAGEN ${ }^{\circledR}$ 2X Multiplex PCR Kit (Qiagen, CA, USA) in a GeneAmp 9700 Thermal Cycler (Applied Biosystems) with an initial denaturation step at $95^{\circ} \mathrm{C}$ for $15 \mathrm{~min}$, followed by 30 cycles of denaturation $\left(94^{\circ} \mathrm{C}\right.$ for $\left.30 \mathrm{~s}\right)$, annealing $\left(57^{\circ} \mathrm{C}\right.$ for $\left.90 \mathrm{~s}\right)$, and extension $\left(72^{\circ} \mathrm{C}\right.$ for $\left.1 \mathrm{~min}\right)$, and a final extension step at $72^{\circ} \mathrm{C}$ for $7 \mathrm{~min}$. The amplified products were diluted in sterile Milli-Q 
Table 1. Description of the lines and control cultivars of the common bean based on VCU tests, including names, types of grains and genealogy composition.

\begin{tabular}{|c|c|c|}
\hline Genotype & Type of grain & Genealogy \\
\hline & & VCU $2003 / 2004$ \\
\hline Carioca 11 & Carioca & Control variety \\
\hline Pérola & Carioca & Control variety \\
\hline CNFC 9435 & Carioca & $\begin{array}{l}\text { A } 769 \text { / } 4 \text { / EMP } 250 \text { /// A } 429 \text { / XAN } 252 \text { // V } 8025 \text { / G } 4449 \text { /// WAF } 2 \text { / A55 // } \\
\text { GN } 31 \text { / XAN } 170\end{array}$ \\
\hline CNFC 9458 & Carioca & EMP 250 / 4 / A 769 /// A 429 / XAN 252 // V 8025 / Pinto UI 114 \\
\hline CNFC 9461 & Carioca & EMP 250 / 4 / A 769 /// A 429 / XAN 252 // V 8025 / Pinto UI 114 \\
\hline CNFC 9471 & Carioca & EMP 250 / 4 / A 769 /// A 429 / XAN 252 // V 8025 / Pinto UI 114 \\
\hline CNFC 9484 & Carioca & EMP 250 / 4 / A 769 /// A 429 / XAN 252 // V 8025 / Pinto UI 114 \\
\hline CNFC 9494 & Carioca & EMP 250 / 4 / A 769 /// A 429 / XAN 252 // V 8025 / Pinto UI 114 \\
\hline CNFC 9500 & Carioca & EMP 250 / 4 / A 769 /// A 429 / XAN 252 // V 8025 / Pinto UI 114 \\
\hline CNFC 9504 & Carioca & EMP 250 / 4 / A 769 /// A 429 / XAN 252 // V 8025 / Pinto UI 114 \\
\hline CNFC 9506 & Carioca & EMP 250 / 4 / A 769 /// A 429 / XAN 252 // V 8025 / Pinto UI 114 \\
\hline CNFC 9518 & Carioca & A $790 /$ A 767 \\
\hline CNFE 8009 & Carioca & PR 17 / ANA 910528 \\
\hline BRS Valente & Black & Control variety \\
\hline CNFP 7966 & Black & CB 733774 / AN 9122526 \\
\hline CNFP 7972 & Black & CB 733774 / AN 9122526 \\
\hline CNFP 7994 & Black & CB 911863 / AN 9123293 \\
\hline CNFP 8000 & Black & CB 911863 / AN 9123293 \\
\hline CNFP 9328 & Black & Xamego / TC 1558-1 \\
\hline CNFP 10138 & Black & CB 911852 / AN 9123293 \\
\hline TB 94-09 & Black & - \\
\hline ТВ $97-13$ & Black & - \\
\hline BRS Vereda & Color: pink & Control variety \\
\hline BRS Radiante & Color: striped & Control variety \\
\hline CNFRJ 10294 & Color: striped & - \\
\hline CNFRJ 10299 & Color: striped & - \\
\hline CNFRX 8035 & Color: purple & BP 9 / MA 721340 // FEB 163 / ARA1 \\
\hline CNFRX 10241 & Color: purple & XAN 283 / RAB 487 / A 247 \\
\hline BRS Marfim & Mulatinho & Control variety \\
\hline CNFM 7957 & Mulatinho & BC 912052 / AN 9022180 \\
\hline CNFM 7958 & Mulatinho & BC 912052 / AN 9022180 \\
\hline CNFM 8057 & Mulatinho & ICA COL 10103 / AN 710988 // Milionário 1732 / LA 721477 \\
\hline CNFM 8080 & Mulatinho & ICA COL 10103 / AN 710988 // LA 721493 / ICA COL 10103 \\
\hline CNFM 9381 & Mulatinho & AN 910518 / A 55 \\
\hline CNFM 9412 & Mulatinho & TC 1558-1 / Aporé \\
\hline CNFM 10375 & Mulatinho & LM 95100047 / SC 9029923 \\
\hline CNFM 10385 & Mulatinho & LM 95100045 / FEB 156 \\
\hline CNFM 10386 & Mulatinho & LM 95100045 / FEB 156 \\
\hline CNFM 10387 & Mulatinho & LM 95100045 / FEB 156 \\
\hline CNFM 10390 & Mulatinho & $\begin{array}{l}\text { LM } 95100045 \text { / FEB } 156 \\
\text { VCU 2003/2004 (additional lines) }\end{array}$ \\
\hline CNFC 7806 & Carioca & Carioca MG // POT 947 / AN910523 \\
\hline CNFC 7813 & Carioca & BZ3836 // FEB 166 / AN 910523 \\
\hline CNFC 8075 & Carioca & LM 21303 / A 248 // LA 721493 / ICA COL 10103 \\
\hline CNFC 8202 & Carioca & EMP 250 / 4 / A 769 /// A 429 / XAN 252 // Pinto UI 114 \\
\hline CNFP 7726 & Black & W22-34 / 84VAN163 \\
\hline CNFP 7762 & Black & W22-34 / VAN163 \\
\hline CNFP 7776 & Black & AN12567 / México 168 \\
\hline CNFP 8104 & Black & Cultivar Corrente submitted to gamma radiation \\
\hline BRS Timbó & Color: purple & Control variety \\
\hline CNFRX 7847 & Color: purple & VCU/color \\
\hline CNFRX 7866 & Color: purple & FEB 163 / AN512879 \\
\hline ARC $1-30$ & - & VCU/weevil \\
\hline ARC $100-4$ & - & VCU/weevil \\
\hline ARC 100T-5 & - & VCU/weevil \\
\hline Talismã & Carioca & Control variety \\
\hline CNFC 9437 & Carioca & EMP 250 / 4 / A769 /// A429 / XAN 252 // V8025 / Pinto UI 114 \\
\hline CNFP 7988 & Black & LM 202203344 / LM 202203330 \\
\hline CNFP 9346 & Black & Xamego / Rudá \\
\hline
\end{tabular}

Continued on next page 
P.C.B. Cardoso et al.

\section{Table 1. Continued.}

\begin{tabular}{|c|c|c|}
\hline Genotype & Type of Grain & Genealogy \\
\hline CNFRJ 10245 & Color: striped & CB 912063 / AN512717 \\
\hline CNFE 8017 & Carioca & PR 9115957 / LR720982CP \\
\hline CNFM 6911 & Mulatinho & BAT $85 \times$ [(A $375 \times$ G 17702) x (A 445 x XAN 112)] \\
\hline \multirow[t]{2}{*}{ CNFM 7119} & Mulatinho & CB511687 / J. PRECOCE \\
\hline & & VCU 2005/2006 \\
\hline BRS Pontal & Carioca & Control variety \\
\hline IAPAR-81 & Carioca & Control variety \\
\hline Magnífico & Carioca & Control variety \\
\hline Pérola & Carioca & Control variety \\
\hline CNFC 10408 & Carioca & $\begin{array}{l}\text { A } 769 \text { / } 4 \text { / A } 774 \text { /// A } 429 \text { / XAN } 252 \text { // V } 8025 \text { / G } 4449 \text { /// WAF 2 / A55 // GN } \\
31 \text { / XAN } 170\end{array}$ \\
\hline CNFC 10410 & Carioca & EMP 205 / 4 / A 769 /// A 429 / XAN 252 // V 8025 / Pinto UI 114 \\
\hline CNFC 10429 & Carioca & EMP 205 / 4 / A 769 /// A 429 / XAN 252 // V 8025 / Pinto UI 114 \\
\hline CNFC 10431 & Carioca & EMP 205 / 4 / A 769 /// A 429 / XAN 252 // V 8025 / Pinto UI 114 \\
\hline CNFC 10432 & Carioca & EMP 205 / 4 / A 769 /// A 429 / XAN 252 // V 8025 / Pinto UI 114 \\
\hline CNFC 10438 & Carioca & EMP 205 / 4 / A 769 /// A 429 / XAN 252 // V 8025 / Pinto UI 114 \\
\hline CNFC 10444 & Carioca & EMP 205 / 4 / A 769 /// A 429 / XAN 252 // V 8025 / Pinto UI 114 \\
\hline CNFC 10455 & Carioca & EMP 205 / 4 / A 769 /// A 429 / XAN 252 // V 8025 / Pinto UI 114 \\
\hline CNFC 10467 & Carioca & AN 51666-0 / LR 720982CP \\
\hline CNFC 10470 & Carioca & PR 9115957 / LR $720982 C P$ \\
\hline BRS Grafite & Black & Control variety \\
\hline BRS Valente & Black & Control variety \\
\hline IPR Uirapuru & Black & Control variety \\
\hline Soberano & Black & Control variety \\
\hline CNFP 10035 & Black & IAPAR BAC 296 // AN 911113 / POT 51 \\
\hline CNFP 10076 & Black & LR 916337 / FE 821732 \\
\hline CNFP 10093 & Black & LA 9017179 / FE 821732 \\
\hline CNFP 10103 & Black & FT 85-113 / POT 51 \\
\hline CNFP 10104 & Black & FT 85-113 / POT 51 \\
\hline CNFP 10109 & Black & FT 85-113 / POT 51 \\
\hline CNFP 10120 & Black & CB 911846 / AN 91232993 \\
\hline CNFP 10206 & Black & $\begin{array}{l}\text { RH5-206 / RAI } 295 \text { /// Honduras 35 / LA } 9017149 \text { // AN } 911113 \text { / CB } 720160 \text { /4/ } \\
\text { AN } 911113 \text { / POT 51 / IAPAR BAC } 296 \text { /// LA9017149 / CB720160 /// FE } 732880\end{array}$ \\
\hline BRS Radiante & Color: striped & Control variety \\
\hline BRS Timbó & Color: purple & Control variety \\
\hline Iraí & Color: manteigão & Control variety \\
\hline Vermelho-2157 & Color: red & Control variety \\
\hline CNFRJ 10559 & Color: striped & PR 95105259 / PR 93203382 \\
\hline CNFRJ 10573 & Color: striped & PR 95105259 / PR 93201759 \\
\hline CNFRJ 10568 & Color: striped & PR 95105259 / PR 93201759 \\
\hline CNFRX 10525 & Color: purple & LM 95105713 / LM 95204049 \\
\hline CNFRX 10527 & Color: purple & LM 95105713 / LM 95204049 \\
\hline CNFRX 10530 & Color: purple & LM 95105713 / LM 95204049 \\
\hline \multirow[t]{2}{*}{ CNFRX 10538} & Color: purple & LM 95105713 / LM 95204049 \\
\hline & & VCU 2007/2008 \\
\hline BRS Cometa & Carioca & Control variety \\
\hline BRS Pontal & Carioca & Control variety \\
\hline IPR Juriti & Carioca & Control variety \\
\hline Pérola & Carioca & Control variety \\
\hline CNFC 10703 & Carioca & LM 95103381 / 95204151 // AN 9022180 / 95204119 /// A 805 \\
\hline CNFC 10713 & Carioca & EMP 250 / 4 / A 769 /// A 429 / XAN 252 // V 8025 / Pinto UI 114 \\
\hline CNFC 10716 & Carioca & EMP 250 / 4 / A 769 /// A 429 / XAN 252 // V 8025 / Pinto UI 114 \\
\hline CNFC 10721 & Carioca & EMP 250 / 4 / A 769 /// A 429 / XAN 252 // V 8025 / Pinto UI 114 \\
\hline CNFC 10729 & Carioca & EMP 250 / 4 / A 769 /// A 429 / XAN 252 // V 8025 / Pinto UI 114 \\
\hline CNFC 10733 & Carioca & EMP 250 / 4 / A 769 /// A 429 / XAN 252 // V 8025 / Pinto UI 114 \\
\hline CNFC 10742 & Carioca & EMP 250 / 4 / A 769 /// A 429 / XAN 252 // V 8025 / Pinto UI 114 \\
\hline CNFC 10753 & Carioca & EMP 250 / 4 / A 769 /// A 429 / XAN 252 // V 8025 / Pinto UI 114 \\
\hline CNFC 10757 & Carioca & EMP 250 / 4 / A 769 /// A 429 / XAN 252 // V 8025 / Pinto UI 114 \\
\hline CNFC 10758 & Carioca & EMP 250 / 4 / A 769 /// A 429 / XAN 252 // V 8025 / Pinto UI 114 \\
\hline CNFC 10762 & Carioca & Pérola / AN 9022180 \\
\hline
\end{tabular}

Continued on next page 


\begin{tabular}{|c|c|c|}
\hline Genotype & Type of Grain & Genealogy \\
\hline $\begin{array}{l}\text { CNFC } 10763 \\
\text { CNFC } 10813 \\
\text { BRS Grafite } \\
\text { BRS Supremo } \\
\text { BRS Valente } \\
\text { IPR Uirapuru } \\
\text { CNFP } 10025 \\
\text { CNFP } 10214 \\
\text { CNFP } 10221 \\
\text { CNFP } 10793 \\
\text { CNFP } 10794 \\
\text { CNFP } 10799 \\
\text { CNFP } 10800 \\
\text { CNFP } 10805 \\
\text { CNFP } 10806 \\
\text { CNFP } 10807\end{array}$ & $\begin{array}{l}\text { Carioca } \\
\text { Carioca } \\
\text { Black } \\
\text { Black } \\
\text { Black } \\
\text { Black } \\
\text { Black } \\
\text { Black } \\
\text { Black } \\
\text { Black } \\
\text { Black } \\
\text { Black } \\
\text { Black } \\
\text { Black } \\
\text { Black } \\
\text { Black }\end{array}$ & $\begin{array}{l}\text { Pérola / AN } 9022180 \\
\text { MAR } 3 \text { /Pérola } \\
\text { Control variety } \\
\text { Control variety } \\
\text { Control variety } \\
\text { Control variety } \\
\text { LA } 9016920 \text { / FE } 821732 \text { // AN } 911113 \\
\text { AN } 730408 \text { / Cornell } 49242 \\
\text { AN } 12567 \text { / LA } 720164 \text { // FE } 821698 \text { / CB } 733795 \\
\text { POT } 51 \text { /// OAC 88-1 / A } 429 \text { // OAC } 88 \text { - 1 / RM } 35 \\
\text { POT } 51 \text { /// ICA Pijao / XAN } 170 \text { /// BAC } 16 \text { / XAN } 91 \\
\text { FT Tarumã / Xamego } \\
\text { FT Tarumã / Xamego } \\
\text { Milionário 1732 / Xamego } \\
\text { Milionário } 1732 \text { / Xamego } \\
\text { IAPAR 44 / Macanudo }\end{array}$ \\
\hline CNFP 10807 & & VCU $2009 / 2010$ \\
\hline $\begin{array}{l}\text { BRS Esplendor } \\
\text { BRS Campeiro } \\
\text { IPR Uirapuru } \\
\text { BRS Supremo } \\
\text { CNFP } 11973 \\
\text { CNFP } 11976 \\
\text { CNFP } 11978 \\
\text { CNFP } 11979 \\
\text { CNFP } 11983 \\
\text { CNFP } 11984 \\
\text { CNFP } 11985 \\
\text { CNFP } 11991 \\
\text { CNFP } 11994 \\
\text { CNFP } 11995 \\
\text { Pérola } \\
\text { BRS Cometa } \\
\text { IPR Juriti } \\
\text { BRS Estilo } \\
\text { CNFE } 8017 \\
\text { CNFC } 11944 \\
\text { CNFC } 11945 \\
\text { CNFC } 11946 \\
\text { CNFC } 11948(P) \\
\text { CNFC } 11951 \\
\text { CNFC } 11952 \\
\text { CNFC } 11953 \\
\text { CNFC } 11954(P) \\
\text { CNFC } 11956 \\
\text { CNFC } 11959 \\
\text { CNFC } 11962 \\
\text { CNFC } 11966(P) \\
\end{array}$ & $\begin{array}{l}\text { Black } \\
\text { Black } \\
\text { Black } \\
\text { Black } \\
\text { Black } \\
\text { Black } \\
\text { Black } \\
\text { Black } \\
\text { Black } \\
\text { Black } \\
\text { Black } \\
\text { Black } \\
\text { Black } \\
\text { Black } \\
\text { Carioca } \\
\text { Carioca } \\
\text { Carioca } \\
\text { Carioca } \\
\text { Carioca } \\
\text { Carioca } \\
\text { Carioca } \\
\text { Carioca } \\
\text { Carioca } \\
\text { Carioca } \\
\text { Carioca } \\
\text { Carioca } \\
\text { Carioca } \\
\text { Carioca } \\
\text { Carioca } \\
\text { Carioca } \\
\text { Carioca } \\
\end{array}$ & 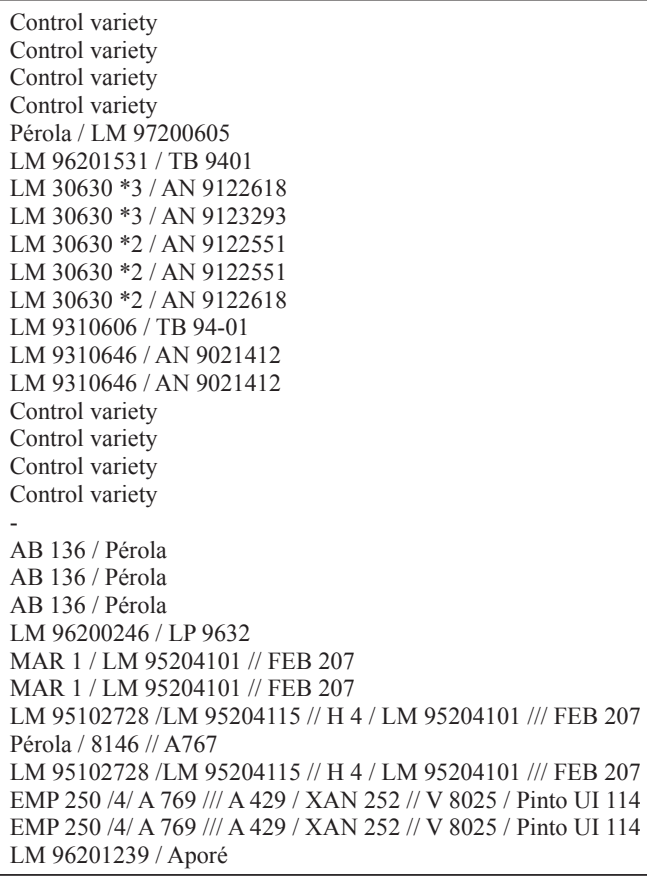 \\
\hline CNFC 11966(P) & & VCU 2011/2012 \\
\hline $\begin{array}{l}\text { CNFP } 15171 \\
\text { CNFP } 15174 \\
\text { CNFP } 15177 \\
\text { CNFP } 15178 \\
\text { CNFP } 15188 \\
\text { CNFP } 15193 \\
\text { CNFP } 15194 \\
\text { CNFP } 15198 \\
\text { CNFP } 15207 \\
\text { CNFP } 15208 \\
\text { IPR Uirapuri }\end{array}$ & $\begin{array}{l}\text { Black } \\
\text { Black } \\
\text { Black } \\
\text { Black } \\
\text { Black } \\
\text { Black } \\
\text { Black } \\
\text { Black } \\
\text { Black } \\
\text { Black } \\
\text { Black }\end{array}$ & $\begin{array}{l}\text { LM 9310639 / LM } 93204217 \\
\text { LM 9310639 / LM } 93204217 \\
\text { LM 9310639 / LM } 93204217 \\
\text { LM 9310639 / LM } 93204217 \\
\text { TB 94-01 / LM 98203893 } \\
\text { TB 94-01 / LM } 98203893 \\
\text { TB 94-01 / LM 98203893 } \\
\text { TB 94-01 / LM 98203893 } \\
\text { TB 94-01 / FPGCF058 } \\
\text { TB 94-01 / FPGCF058 } \\
\text { Control variety }\end{array}$ \\
\hline
\end{tabular}

Continued on next page 


\begin{tabular}{|c|c|c|}
\hline Genotype & Type of Grain & Genealogy \\
\hline BRS Campeiro & Black & Control variety \\
\hline BRS Esplendor & Black & Control variety \\
\hline CNFC 15001 & Carioca & $\begin{array}{l}\text { RS*: Aporé, BAT 477, AN 9022180, Corrente, A 806, MAN 48, AND 277, IPA 7, } \\
\text { A797, Pinto Villa, EMP 81, V 8025, A 525, A 774, MAR } 1 \text { and DOR 500 }\end{array}$ \\
\hline CNFC 15003 & Carioca & $\begin{array}{l}\text { RS: Aporé, BAT 477, AN 9022180, Corrente, A 806, MAN 48, AND 277, IPA 7, } \\
\text { A797, Pinto Villa, EMP 81, V 8025, A 525, A 774, MAR } 1 \text { and DOR } 500\end{array}$ \\
\hline CNFC 15010 & Carioca & $\begin{array}{l}\text { RS: Aporé, BAT 477, AN 9022180, Corrente, A 806, MAN 48, AND 277, IPA 7, } \\
\text { A797, Pinto Villa, EMP 81, V 8025, A 525, A 774, MAR } 1 \text { and DOR 500 }\end{array}$ \\
\hline CNFC 15018 & Carioca & $\begin{array}{l}\text { RS: Aporé, BAT 477, AN 9022180, Corrente, A 806, MAN 48, AND 277, IPA 7, } \\
\text { A797, Pinto Villa, EMP 81, V 8025, A 525, A 774, MAR } 1 \text { and DOR 500 }\end{array}$ \\
\hline CNFC 15023 & Carioca & $\begin{array}{l}\text { RS: Aporé, BAT 477, AN 9022180, Corrente, A 806, MAN 48, AND 277, IPA 7, } \\
\text { A797, Pinto Villa, EMP 81, V 8025, A 525, A 774, MAR } 1 \text { and DOR 500 }\end{array}$ \\
\hline CNFC 15025 & Carioca & $\begin{array}{l}\text { RS: Aporé, BAT 477, AN 9022180, Corrente, A 806, MAN 48, AND 277, IPA 7, } \\
\text { A797, Pinto Villa, EMP 81, V 8025, A 525, A 774, MAR } 1 \text { and DOR 500 }\end{array}$ \\
\hline CNFC 15033 & Carioca & $\begin{array}{l}\text { RS: Aporé, BAT 477, AN 9022180, Corrente, A 806, MAN 48, AND 277, IPA 7, } \\
\text { A797, Pinto Villa, EMP 81, V 8025, A 525, A 774, MAR } 1 \text { and DOR } 500\end{array}$ \\
\hline CNFC 15035 & Carioca & $\begin{array}{l}\text { RS: Aporé, BAT 477, AN 9022180, Corrente, A 806, MAN 48, AND 277, IPA 7, } \\
\text { A797, Pinto Villa, EMP 81, V 8025, A 525, A 774, MAR } 1 \text { and DOR } 500\end{array}$ \\
\hline CNFC 15038 & Carioca & $\begin{array}{l}\text { RS: Aporé, BAT 477, AN 9022180, Corrente, A 806, MAN 48, AND 277, IPA 7, } \\
\text { A797, Pinto Villa, EMP 81, V 8025, A 525, A 774, MAR } 1 \text { and DOR 500 }\end{array}$ \\
\hline CNFC 15044 & Carioca & $\begin{array}{l}\text { RS: Aporé, BAT 477, AN 9022180, Corrente, A 806, MAN 48, AND 277, IPA 7, } \\
\text { A797, Pinto Villa, EMP 81, V 8025, A 525, A 774, MAR } 1 \text { and DOR 500 }\end{array}$ \\
\hline CNFC 15049 & Carioca & $\begin{array}{l}\text { RS: Aporé, BAT 477, AN 9022180, Corrente, A 806, MAN 48, AND 277, IPA 7, } \\
\text { A797, Pinto Villa, EMP 81, V 8025, A 525, A 774, MAR } 1 \text { and DOR 500 }\end{array}$ \\
\hline CNFC 15070 & Carioca & $\begin{array}{l}\text { RS: Aporé, BAT 477, AN 9022180, Corrente, A 806, MAN 48, AND 277, IPA 7, } \\
\text { A797, Pinto Villa, EMP 81, V 8025, A 525, A 774, MAR } 1 \text { and DOR 500 }\end{array}$ \\
\hline CNFC 15082 & Carioca & $\begin{array}{l}\text { RS: Aporé, BAT 477, AN 9022180, Corrente, A 806, MAN 48, AND 277, IPA 7, } \\
\text { A797, Pinto Villa, EMP 81, V 8025, A 525, A 774, MAR } 1 \text { and DOR 500 }\end{array}$ \\
\hline CNFC 15086 & Carioca & $\begin{array}{l}\text { RS: Aporé, BAT 477, AN 9022180, Corrente, A 806, MAN 48, AND 277, IPA 7, } \\
\text { A797, Pinto Villa, EMP 81, V 8025, A 525, A 774, MAR } 1 \text { and DOR 500 }\end{array}$ \\
\hline CNFC 15097 & Carioca & OXI-16 \\
\hline BRS Estilo & Carioca & Control variety \\
\hline Pérola & Carioca & Control variety \\
\hline
\end{tabular}

$* \mathrm{RS}=$ recurrent selection.

$\mathrm{H}_{2} \mathrm{O}$ in proportions ranging from 1:5 to $1: 10$ depending on the concentration of the amplified products. Electrophoresis was performed using a mixture containing $0.5 \mu \mathrm{L}$ diluted amplification product, $0.08 \mu \mathrm{L} 500$ ROX size standard (Applied Biosystems), and $9.42 \mu \mathrm{L}$ formamide Hi-Di (Applied Biosystems). The electrophoresis was performed on an ABI3100 platform (Applied Biosystems) using filter " $\mathrm{D}$ " for fluorescence reading. The analysis of the fragments was performed using GeneScan Analysis 2.1 (Applied Biosystems), and the sizes of the alleles were obtained using the GeneMapper 3.5 program (Applied Biosystems).

\section{Statistical analyses}

The genetic analysis was conducted based on the molecular profiles generated from the allele frequencies of polymorphic loci, expected heterozygosity $\left(H_{\mathrm{E}}\right)$, and observed heterozygosity $\left(H_{\mathrm{O}}\right)$, according to Nei (1987). The number of private alleles was determined using the Genetic Data Analysis software (Lewis and Zaykin, 2001). The genetic distances were estimated by the Rogers coefficient modified by Wright using the NTSYS v.2.02 soft- 
ware (Rohlf, 1993) and then subjected to cluster analysis using the UPGMA algorithm. The data were plotted on a dendrogram for the visualization of genetic distances. The estimated power of exclusion, probability of identity, and number of identical individuals were calculated using the Identity v.1.0 software (Wagner and Sefc, 1999). Wright F-statistic was calculated with 10,000 bootstrap resamplings using the FSTAT v.2.9.3.2 software (Goudet, 2002). The factorial correspondence analysis (FCA) was obtained using the GENETIX v.4.03 software (Belkhir et al., 2004). Structure analysis was performed using the STRUCTURE v.2.2 software (Pritchard et al., 2000; Falush et al., 2003). The $\Delta \mathrm{K}$ statistic (Evanno et al., 2005) was used to determine the number of genetically homogeneous groups using the Structure software (Earl and VonHold, 2011).

Table 2. Genotyping panels composed of 36 microsatellite markers used for the molecular characterization of common bean cultivars, including the name of the loci, their respective fluorescence, amplification range in bp, annealing temperature, amplification patterns, chromosomes and references.

\begin{tabular}{|c|c|c|c|c|c|c|}
\hline Panel & Marker & Fluorescence & Size range (bp) & Amplification pattern & Chromosome & References \\
\hline \multirow[t]{4}{*}{1} & BM185* & HEX & $97-114$ & Specific & 4 & Gaitán-Solís et al. (2002) \\
\hline & BM138 & NED & $196-202$ & Specific & 7 & Gaitán-Solís et al. (2002) \\
\hline & BM183* & 6-FAM & $142-156$ & Specific & 4 & Gaitán-Solís et al. (2002) \\
\hline & PvBR163* & 6-FAM & $210-330$ & Specific** & 1 & Grisi et al. (2007) \\
\hline \multirow[t]{3}{*}{2} & BM143* & HEX & $110-168$ & Specific & 9 & Gaitán-Solís et al. (2002) \\
\hline & BM212* & NED & $194-210$ & Specific & 8 & Gaitán-Solís et al. (2002) \\
\hline & PvBR025* & 6-FAM & $153-177$ & Specific** & 9 & Buso et al. (2006) \\
\hline \multirow[t]{3}{*}{3} & PvBR005* & HEX & $168-193$ & Specific & 1 & Buso et al. (2006) \\
\hline & BM164 & NED & $147-182$ & Specific & 9 & Gaitán-Solís et al. (2002) \\
\hline & PvBR35 & 6-FAM & $204-250$ & Specific & 4 & Grisi et al. (2007) \\
\hline \multirow[t]{5}{*}{4} & BM155 & HEX & $107-111$ & Specific & 7 & Gaitán-Solís et al. (2002) \\
\hline & PvBR168 & HEX & $180-185$ & Specific & 11 & Grisi et al. (2007) \\
\hline & PvBR087* & NED & $150-188$ & Specific** & 5 & Grisi et al. (2007) \\
\hline & BM140 & 6-FAM & $157-175$ & Specific & 10 & Gaitán-Solís et al. (2002) \\
\hline & BM114* & 6-FAM & $209-261$ & Specific & 11 & Gaitán-Solís et al. (2002) \\
\hline \multirow[t]{4}{*}{5} & BM187* & HEX & $162-267$ & Specific** & 1 & Gaitán-Solís et al. (2002) \\
\hline & PvBR113* & NED & $74-100$ & Specific & 6 & Grisi et al. (2007) \\
\hline & BM181* & NED & $182-190$ & Specific & 5 & Gaitán-Solís et al. (2002) \\
\hline & PvBR053 & 6-FAM & $165-171$ & Specific & 3 & Grisi et al. (2007) \\
\hline \multirow[t]{4}{*}{6} & PvBR012 & HEX & $188-193$ & Specific & 8 & Buso et al. (2006) \\
\hline & BM068 & NED & $169-171$ & Specific & 10 & Gaitán-Solís et al. (2002) \\
\hline & BM175 & 6-FAM & $155-188$ & Specific & 7 & Gaitán-Solís et al. (2002) \\
\hline & BM149 & 6-FAM & $245-255$ & Specific & 10 & Gaitán-Solís et al. (2002) \\
\hline \multirow[t]{5}{*}{7} & BM165* & HEX & $170-187$ & Specific & 3 & Gaitán-Solís et al. (2002) \\
\hline & PvBR215 & NED & 220 & Specific & 6 & Grisi et al. (2007) \\
\hline & BM202* & 6-FAM & $134-156$ & Specific** & 11 & Gaitán-Solís et al. (2002) \\
\hline & BM154* & 6-FAM & $160-307$ & Specific** & 11 & Gaitán-Solís et al. (2002) \\
\hline & BM211* & 6-FAM & $181-242$ & Specific** & 3 & Gaitán-Solís et al. (2002) \\
\hline \multirow[t]{2}{*}{8} & BM201* & NED & $96-114$ & Specific & 2 & Gaitán-Solís et al. (2002) \\
\hline & PvBR251 & HEX & 203-205 & Specific & 2 & Grisi et al. (2007) \\
\hline \multirow[t]{6}{*}{9} & PvBR011* & HEX & $182-190$ & Specific & 9 & Buso et al. (2006) \\
\hline & PvBR169* & HEX & $204-212$ & Specific & 6 & Grisi et al. (2007) \\
\hline & BM210* & NED & $166-188$ & Specific** & 4 & Gaitán-Solís et al. (2002) \\
\hline & BM189* & NED & $102-112$ & Specific & 5 & Gaitán-Solís et al. (2002) \\
\hline & PvBR013* & 6-FAM & $169-195$ & Specific & 1 & Grisi et al. (2007) \\
\hline & PvBR272* & 6-FAM & $78-116$ & Specific** & 11 & Grisi et al. (2007) \\
\hline
\end{tabular}

*Polymorphic markers used to derive the genetic parameters for analysis. **Markers that showed interpretable PCR products, although they contained some nonspecific bands. 


\section{RESULTS AND DISCUSSION}

\section{Characterization of microsatellite markers}

Recently, the use of microsatellite markers for individual genotyping has evolved for the development of semi-automated, multilocus genotyping systems due to the increase in automation and efficiency of the reagents for molecular analysis. Over the years, the goal has been to develop universal and robust genetic identification systems to analyze genetic relationships individual identification, population structure, and genetic diversity. The nine multiplex systems used in the present study consisted of 36 microsatellite markers exhibiting no evidence of linkage and were widely distributed across 11 chromosomes in the bean genome according to genetic map information (Grisi et al., 2007; Garcia et al., 2011). The number of marker sets per system ranged from 3 to 6 , with an average of 4 markers per panel. The same amplification conditions were used, which allowed for fast, efficient, and simultaneous amplification of the loci. Based on tri-nucleotide repetition, all markers, with the exception of marker BM149, were derived from di-nucleotide repeats with alleles that differ by $2 \mathrm{bp}$, which is the type of marker currently used for identification purposes in P. vulgaris. Despite the inherent limitations of analysis based on di-nucleotides, such as difficulties in interpretation due to stuttering and low detection power for alleles that differ by a few base pairs even when using high-resolution electrophoresis, genotyping based on di-nucleotide repeats still predominates among plants due to their high abundance and ease of identification in the genomes. More recently, because of efforts in genomic sequencing and the availability of expressed sequence tag databanks, a greater number of tri-nucleotide markers are being developed (Blair et al., 2011).

Of the 36 markers used in the VCU analysis, 24 were polymorphic for the five tests. The total number of identified alleles was 187, varying from 3 to 18 , with an average of 7.79 alleles per locus. The average $H_{\mathrm{E}}$ identified among loci was $65 \%$, the largest $H_{\mathrm{E}}$ was identified at locus BM154 (88\%) and the lowest $H_{\mathrm{E}}$ at the BM212 locus (23\%) (Table 3). The $H_{\mathrm{O}}$, which is a genetic diversity index influenced by the reproductive system of the species, ranged from 0 to 0.045 , with an average of 0.013 , due to the self-pollination process for obtaining new grains or seeds. Studies on cross-pollination of the common bean involving wild, cultivated (Zizumbo-Villarreal et al., 2005; Ferreira et al., 2007), or transgenic germplasm (Faria et al., 2010) have reported similar results, with outcrossing rates below $2 \%$ and slight variations depending on the cultivar and environmental conditions.

The data also allowed for a comparison of results between laboratories based on common markers previously characterized in diverse sources of germplasm accessions, such as all the markers belonging to the "BM" series and some markers belonging to the "PvBr" (PvBr35, PvBr87, PvBr163, PvBr243) series. Overall, the estimates of genetic diversity based on microsatellite markers described in other studies have been small compared to the set of markers used in the present study. Benchimol et al. (2007) characterized varieties of the common bean with a set of 87 di-nucleotide SSRs in two of the major gene pools (Andean and Mesoamerican). These authors observed an average number of alleles of 2.82 and an average PIC of 0.45 , significantly lower than those found in the present study (7.79 and 0.65 , respectively). Similarly, Métais et al. (2002) reported an average PIC of 0.44 for 15 markers based on the analysis of 45 common bean lines representing nine classes of 
grains. Recently, studies by Blair et al. (2009) using the cultivated and wild germplasms of Andean and Mesoamerican origins revealed close PIC estimates for di- $(0.48)$ and trinucleotides (0.40).

Table 3. Genetic descriptors of 24 microsatellite loci of the common beans analyzed, including the number of alleles per locus $\left(N_{\mathrm{A}}\right)$, expected heterozygosity $\left(H_{\mathrm{E}}\right)$, observed heterozygosity $\left(H_{\mathrm{O}}\right)$, probability of exclusion $(\mathrm{PE})$ and probability of identity $(\mathrm{PI})$.

\begin{tabular}{|c|c|c|c|c|c|c|}
\hline Locus & Size range (bp) & $N_{\mathrm{A}}$ & $H_{\mathrm{E}}$ & $H_{\mathrm{O}}$ & $\mathrm{PE}$ & PI \\
\hline BM114 & $209-261$ & 7 & 0.583 & 0.022 & 0.353 & 0.303 \\
\hline BM143 & $110-168$ & 9 & 0.819 & 0.006 & 0.636 & 0.109 \\
\hline BM154 & $160-307$ & 17 & 0.876 & 0.037 & 0.752 & 0.051 \\
\hline BM165 & $170-187$ & 5 & 0.465 & 0.017 & 0.212 & 0.514 \\
\hline BM181 & $182-190$ & 3 & 0.306 & 0.000 & 0.141 & 0.589 \\
\hline BM183 & $142-156$ & 6 & 0.575 & 0.006 & 0.352 & 0.300 \\
\hline BM185 & $97-114$ & 7 & 0.764 & 0.034 & 0.550 & 0.161 \\
\hline BM187 & $162-267$ & 11 & 0.745 & 0.026 & 0.533 & 0.167 \\
\hline BM189 & $102-112$ & 5 & 0.610 & 0.006 & 0.334 & 0.378 \\
\hline BM201 & $96-114$ & 7 & 0.756 & 0.045 & 0.537 & 0.172 \\
\hline BM202 & $134-156$ & 5 & 0.605 & 0.013 & 0.313 & 0.410 \\
\hline BM210 & $168-188$ & 9 & 0.635 & 0.017 & 0.367 & 0.342 \\
\hline BM212 & $194-210$ & 3 & 0.230 & 0.000 & 0.108 & 0.655 \\
\hline PvBr005 & $168-193$ & 8 & 0.814 & 0.044 & 0.626 & 0.115 \\
\hline PvBr011 & $182-190$ & 3 & 0.478 & 0.000 & 0.198 & 0.569 \\
\hline PvBr013 & $169-195$ & 7 & 0.815 & 0.028 & 0.626 & 0.115 \\
\hline PvBr025 & $153-177$ & 11 & 0.771 & 0.011 & 0.574 & 0.145 \\
\hline PvBr035 & $204-250$ & 5 & 0.582 & 0.006 & 0.290 & 0.446 \\
\hline PvBr087 & $150-188$ & 8 & 0.636 & 0.000 & 0.389 & 0.295 \\
\hline PvBr113 & 74-100 & 8 & 0.724 & 0.022 & 0.498 & 0.201 \\
\hline PvBr163 & $210-330$ & 18 & 0.839 & 0.025 & 0.701 & 0.066 \\
\hline PvBr169 & $204-212$ & 3 & 0.406 & 0.035 & 0.170 & 0.573 \\
\hline PvBr243 & $221-255$ & 10 & 0.832 & 0.018 & 0.674 & 0.085 \\
\hline PvBr272 & $78-116$ & 12 & 0.873 & 0.006 & 0.739 & 0.058 \\
\hline Average & - & 7.79 & 0.656 & 0.013 & - & - \\
\hline Total & & 187 & - & - & 0.999 & $1.32 \times 10^{-16}$ \\
\hline
\end{tabular}

In the present study, the markers selected for the genotyping panels also had high discriminatory power, which was confirmed by the high power of exclusion (0.999) and low values of combined probability of identity $\left(1.32 \times 10^{-16}\right)$. Thus, the probability of identifying two individuals with the same genotype in a population using this set of markers is extremely low (Table 3). However, even with the high power of discrimination among accessions by microsatellite markers used in the present study and blind experiments for genetic analysis, where the ratio of genetic relationships among the samples was not known, the results indicated the presence of identical genotypes within and between VCU tests. This result was due to the selection of similar lines for testing. The presence of identical genotypes could be avoided by tighter control of the pedigree or by using the microsatellite characterization results as a criterion to choose the accessions that will be part of the VCU tests.

To identify the genetically indistinguishable lines, new genotyping was performed using bulk decomposition and the criterion of at least two genetic differences to declare two materials as distinct from each other with high reliability. The identical genotypes included the carioca lines CNFC 9500 and CNFC 9494 from VCU 2003/2004 and the line CNFC 10444 from VCU 2004/2005, all of which were derived from the same cross (i.e., EMP 250 / 4 / A 769 /// A 429 / $252 \mathrm{XAN} / / \mathrm{V} 8025 \mathrm{~V} /$ Pinto UI 114). The same result was observed for the black grain lines, CNFP 10805 and CNFP 10806, which were derived from the Millionaire 1732/Xamego cross. 
The largest number of lines sharing the same molecular profile was observed in the 2011/2012 VCU test, where 12 lines exhibited total genetic identity with at least one line within the test itself. Similarly, the analysis allowed for the identification of genetically divergent lines between the selection cycles, which can drive the development of populations with wider genetic basis, thereby increasing the possibility of new allele combinations from accessions selected by breeders for the characteristics considered important.

\section{Molecular characterization of VCU tests}

A total of 150 lines integrating the five VCU tests were characterized. In addition, the estimates of genetic parameters per test and estimates based on the analysis of 24 common microsatellite loci were described (Table 4). A total of 145 alleles were identified in the $2003 / 2004$ VCU test, and the average $H_{\mathrm{E}}$ among accessions was 0.50 . The highest $H_{\mathrm{E}}$ was identified for the color group (0.63), and the lowest was observed in the weevil group (0.24). The highest estimates of genetic diversity obtained for the four remaining VCU tests were due to the more diverse composition of common bean accessions, because they are composed of five distinct grain classes. Similarly, the 2005/2006 VCU test, which had the second highest estimate of $H_{\mathrm{E}}(0.49)$, also included a test called "VCU Colors", which comprises three different types of beans (striped, purple, and pinkish).

\begin{tabular}{|c|c|c|c|c|c|c|}
\hline $\mathrm{VCU}$ & Type & Accessions & Alleles & $H_{\mathrm{E}}$ & $H_{\mathrm{O}}$ & $F_{\mathrm{ST}}$ \\
\hline \multirow[t]{6}{*}{ VCU 2003/04 } & Carioca & 17 & 103 & 0.571 & 0.020 & \multirow{6}{*}{$\begin{array}{l}0.122 \\
95 \% \mathrm{CI}=0.083-0.163\end{array}$} \\
\hline & Black & 14 & 85 & 0.499 & 0.018 & \\
\hline & Colors & 07 & 78 & 0.630 & 0.079 & \\
\hline & Mulatinho & 13 & 86 & 0.576 & 0.008 & \\
\hline & Weevil & 3 & 35 & 0.236 & 0.014 & \\
\hline & & 54 & 145 & 0.502 & 0.028 & \\
\hline \multirow[t]{4}{*}{ VCU 2005/06 } & Carioca & 10 & 62 & 0.402 & 0.083 & \multirow{4}{*}{$\begin{array}{l}0.259 \\
95 \% \mathrm{CI}=0.184-0.336\end{array}$} \\
\hline & Black & 8 & 64 & 0.495 & 0.000 & \\
\hline & Colors & 7 & 67 & 0.580 & 0.080 & \\
\hline & & 25 & 122 & 0.492 & 0.029 & \\
\hline \multirow{3}{*}{ VCU 2007/08 } & Carioca & 13 & 64 & 0.466 & 0.000 & \\
\hline & Black & 10 & 66 & 0.443 & 0.000 & \multirow[t]{2}{*}{$95 \% \mathrm{CI}=0.194-0.380$} \\
\hline & & 23 & 93 & 0.455 & 0.000 & \\
\hline \multirow[t]{3}{*}{ VCU 2009/10 } & Carioca & 13 & 84 & 0.497 & 0.024 & \multirow{3}{*}{$\begin{array}{l}0.190 \\
95 \% \mathrm{CI}=0.123-0.256\end{array}$} \\
\hline & Black & 10 & 64 & 0.408 & 0.041 & \\
\hline & & 23 & 102 & 0.453 & 0.033 & \\
\hline \multirow[t]{3}{*}{ VCU 2011/12 } & Carioca & 15 & 62 & 0.314 & 0.000 & \multirow{3}{*}{$\begin{array}{l}0.415 \\
95 \% \mathrm{CI}=0.328-0.501\end{array}$} \\
\hline & Black & 10 & 54 & 0.394 & 0.000 & \\
\hline & & 25 & 83 & 0.354 & 0.000 & \\
\hline \multicolumn{2}{|l|}{ Average } & - & - & 0.451 & 0.018 & \multirow{2}{*}{$\begin{array}{l}0.159^{*} \\
95 \% \mathrm{CI}=0.100-0.227\end{array}$} \\
\hline Total & & 150 & 189 & - & - & \\
\hline
\end{tabular}

*VCU that is significantly different from zero $(\mathrm{P}<0.05) . H_{\mathrm{E}}=$ expected heterozygosity; $H_{\mathrm{O}}=$ observed heterozygosity; $F_{\mathrm{ST}}=$ average inbreeding coefficient; $95 \% \mathrm{CI}=95 \%$ confidence interval.

Considering only the estimates derived from the classes of grains, such as black and carioca, the 2003/2004 VCU test exhibited the highest $H_{\mathrm{E}}$ value $(0.53)$, followed by the $2005 / 2006,2007 / 2008$, and 2009/2010 VCU tests, with estimates close to 0.45 ; the lowest value was observed in the $2011 / 2012$ test, with an $H_{\mathrm{E}}$ value of 0.35 . Because the $2003 / 2004$ 
and 2011/2012 VCU tests were performed with a similar number of lines (31 and 25, respectively), the reduction in $H_{\mathrm{E}}$ could have been attributed to the loss of genetic diversity caused by the selection of lines that were more similar, which was evidenced in the most recent VCU test (2011/2012). Particularly for tests performed with the carioca grain, the total $H_{\mathrm{E}}$ was reduced by approximately $50 \%$ and ranged from 0.57 to 0.31 for the $2003 / 2004$ and $2011 / 2012 \mathrm{VCU}$ tests, respectively. Similarly, the average number of alleles was reduced from 7.8 (2002/2003 VCU) to 4.13 (2011/2012 VCU). The diversity reduction in breeding programs indicates a narrowing of the genetic base upon which the lines are selected. This reduction may be due to the use of a limited number of parents, genetically related parents, and/or a frequent occurrence of the same ancestors. These results can drastically reduce the short-term genetic gain for agronomically important traits such as yield. The economic impact will be greater for cultivars of carioca grain, which currently represents $80 \%$ of the national preference, since only the variability enables the imposition of the selection process (Ramalho et al., 2004).

Several authors working in major research institutions in Brazil have reported estimates of genetic genetic gain for the culture of the common bean, especially for cultivars such as carioca. In general, the relative average progress is approximately 1.21 to $1.90 \%$ per year, representing absolute gains of approximately $14-30 \mathrm{~kg} / \mathrm{ha} /$ year (Abreu et al., 2004). Recently, Matos (2005) reported gains of only $0.6 \%$ per year after evaluating the period from 1974 to 2004. According to Chiorato (2008), since 1997, when the use of recommended controls registered in MAPA (2011) in the VCU tests became mandatory, the relative productivity gains tended to be lower due to the difficulty of developing more productive genotypes than controls, even though new attributes, such as grain quality and disease resistance, are used as differentiators.

Based on the estimates of allele frequency, $16.6 \%$ of the alleles displayed a frequency of $\leq 5 \%$ and only $0.5 \%$ had a frequency of $\leq 1 \%$. Of the 189 alleles identified using 24 microsatellites, $48(26 \%)$ corresponded to private alleles (exclusive alleles), and the vast majority $(65 \%)$ occurred at frequencies below $5 \%$. A total of 15 lines $(10 \%$ of the total) displayed exclusive alleles. The groups of grains with the highest number of private alleles were black ( 7 alleles) and carioca (6 alleles), which occurred in 12 of the 24 loci used in the analysis. These data are relevant because they allow for the selection of lines that have more divergent alleles to incorporate agronomically rare alleles and contribute to the expansion of genetic diversity in the pool of cultivated germplasm.

The estimates of genetic distance obtained from the VCU tests and the total pool of genotypes characterized are both important findings of the data analysis (Figure 1, Panels A and B). Among the five trials, the average genetic distance was 0.34 . The smallest distance was that from the $2003 / 2004$ and $2005 / 2006$ VCUs $(0.23)$ and the highest was that from the 2003/2004 and 2011/2012 VCUs (0.44), with a cophenetic correlation coefficient (r) of 0.97 $(\mathrm{P} \leq 0.031)$. The more recent VCUs (2009/2010 and 2011/12) also displayed a low genetic distance $(0.26)$, which is a troubling indication that the lines comprising each cycle of VCU are derived from genetically close groups. Based on the line and grain type, the smallest genetic distances were obtained between the carioca and black grains $(0.32)$ and the highest among the black and weevil (0.53) groups, with an average of 0.41 and an index cophenetic correlation (r) of $0.98(\mathrm{P} \leq 0.031)$.

The distribution of the groups through spatial structure can be best visualized by the FCA illustrated in Figure 2. The establishment of a pre-breeding program, in which the cultivated germplasm serves as a source of genetic variability, is reported as a strategy to increase 
the genetic base of cultivars (Rangel et al., 1996). A molecular characterization of 50 cultivars of $P$. vulgaris performed at different institutions (Cardoso, 2008) revealed an average genetic distance of $0.72(\mathrm{r}=0.96)$, indicating that there is satisfactory genetic differentiation between materials. Thus, the recurrent use of these divergent genotypes with adequate characteristics can contribute effectively to increase the genetic base of the breeding program.
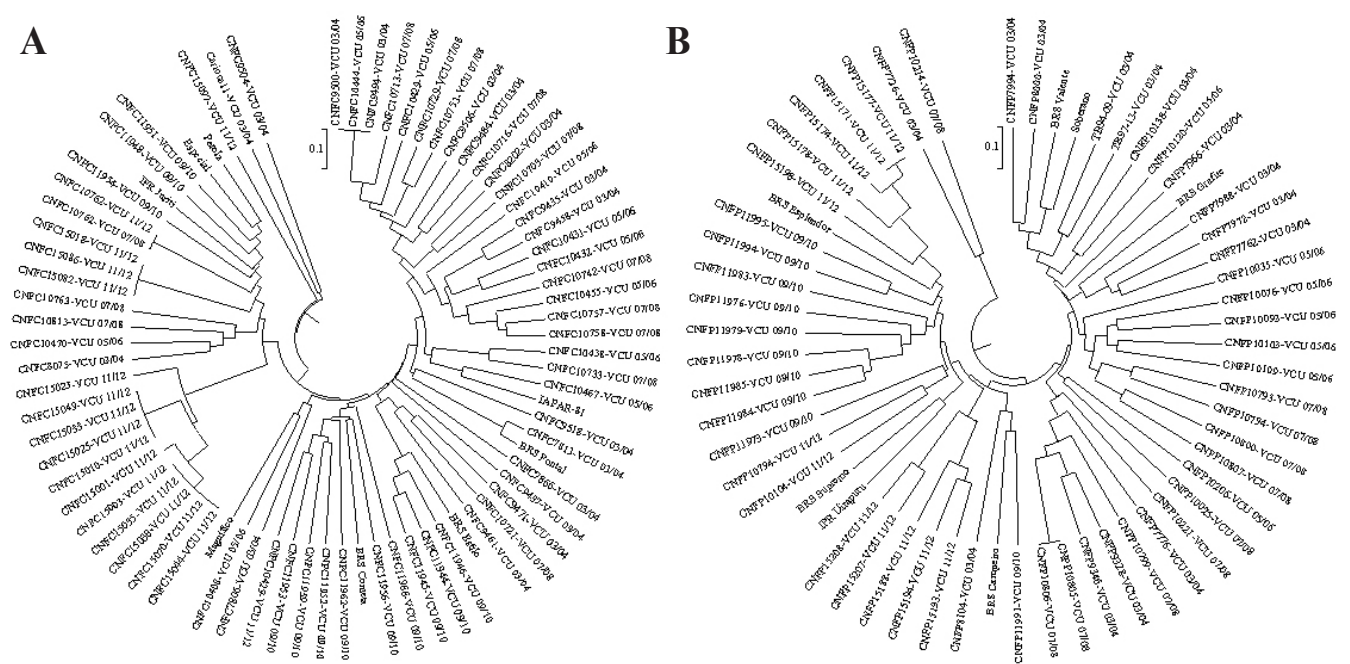

Figure 1. Dendrogram displaying the genetic distances between the carioca (Panel A) and black grain (Panel B) lines and controls based on the average genetic distance of Rogers' coefficient modified by Wright $(\mathrm{P}=0.002 ; 1000$ permutations).

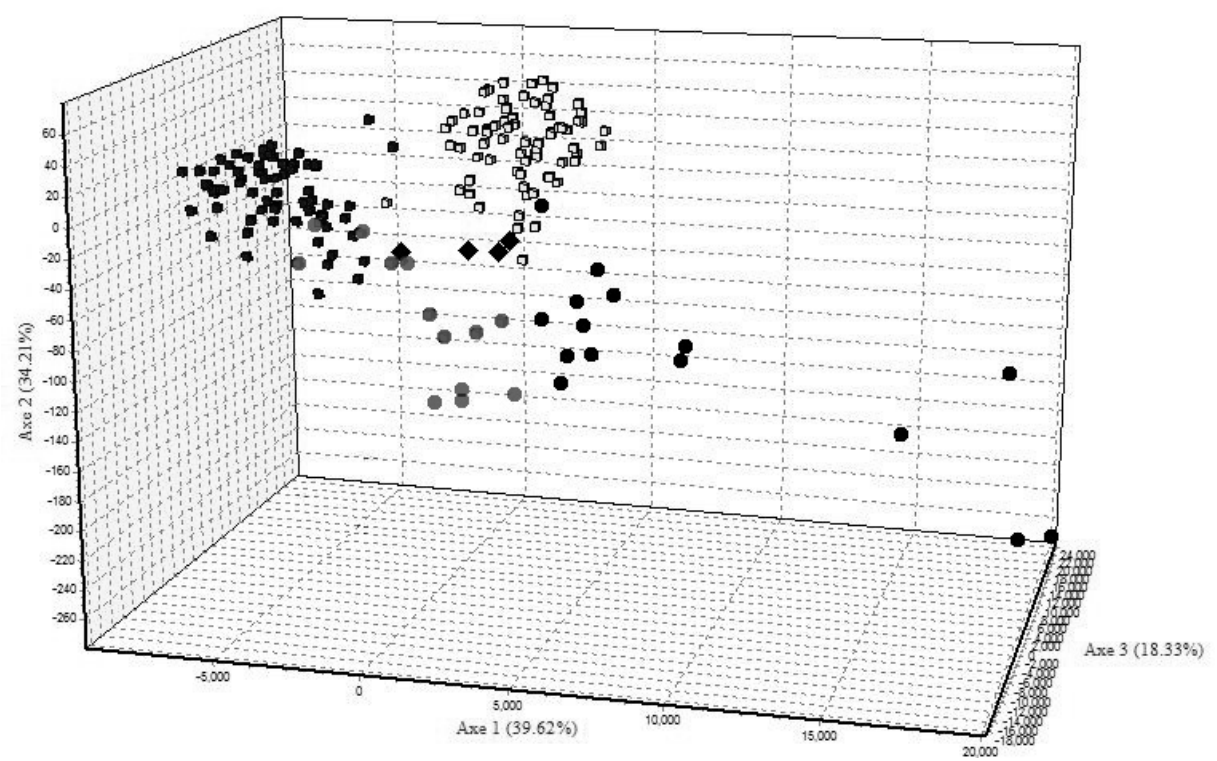

Figure 2. FCA and distribution pattern of genetic variability among the different line groups that are part of the VCU analysis: carioca group (white squares), black group (black squares), mulatinho group (gray circles), weevil group (diamonds), and VCU/color (black circles). 
Applying Wright F-statistic to estimate genetic structure (VCU test) (Table 4), the inbreeding coefficient $\left(F_{\mathrm{ST}}\right)$ values indicated a high degree of differentiation in the tests evaluated, with indices ranging from 0.122 (VCU 2003/2004) to 0.415 (VCU 2011/2012). However, the inbreeding coefficient values decreased between tests of the same grain type due to the frequent occurrence of the same ancestors in the genealogy of the studied lines (Table 1). The average $F_{\mathrm{ST}}$ value estimated between the five VCU tests was 0.159 , indicating genetic differentiation among the line groups tested. This differentiation could be attributed to the selection of differentiated progenitors that represent the specific tests for each type of grain. This result occurs because the parents that are used for crossing and/ or backcrossing (obtaining genetic variability for exploitation in the breeding program) are selected based on Brazilian regions targeted by the program and for characteristics such as productivity, adaptation, and resistance/tolerance to pests and diseases, as well as other specific characteristics. The largest structure was observed $\left(F_{\mathrm{ST}}=0.415\right)$ between the groups of black and carioca grains in the 2011/2012 VCU test. These estimates are consistent with those obtained using the method for obtaining lines. The black grain lines were derived from bi-parental crosses composed of only five parents, and the carioca grain lines were obtained by recurrent selection consisting of 14 parents, resulting in higher differentiation between groups.

In addition, Bayesian clustering analysis performed using the STRUCTURE software resulted in the formation of two distinct groups $(K=2)$. This division can be attributed to the genotypes that comprise the population base of the breeding program. The lines of the three VCUs conducted from 2003 to 2008 share part of the genealogy of the different groups of grains characterized, and the same result is found between the last two tests (2009-2012). This structure is explained when the pedigrees of the materials are verified, corresponding to line members of the VCU tests over the different periods. The blocks of crosses performed resulted in a large number of lines with characteristics that are interesting for the breeding programs, and, over the years, these lines were evaluated for agronomic performance using VCU tests. Lines derived from the same initial crossing block can constitute consecutive VCU tests due to the program pressure to maintain target characteristics over a number of years. When lines share the same ancestry and the potential to evaluate the VCUs is not available, lines derived from new crosses are developed and integrated into subsequent VCUs. Thus, VCUs conducted in consecutive years tend to group according to their genealogy, and therefore, sharing of the same genetic structure is expected.

\section{CONCLUSIONS}

Genetic diversity analysis from VCU testing using molecular markers generated relevant data for the common bean breeding program, such as determining and monitoring the level of genetic variation between groups of lines within and between tests and the identification of genotypes that are genetically indistinguishable. Consequently, these genetically indistinguishable lines can be eliminated from the tests. These findings lead to direct cost reductions and to the identification of the molecular profile of each strain, thereby constituting an innovative tool for genetic diversity analysis of the cultivar of the common bean for the process of varietal protection. The set of di-nucleotide loci used in the present 
study resulted in the establishment of a genetic analysis system for the common bean with high resolving power. This system currently integrates and complements the routine activities of the program, thus constituting effective molecular tools to characterize and distinguish bean lines. The fact that the carioca and black bean commercial lines have reduced genetic variability indicates the need for the inclusion of parents with a larger genetic base in the blocks of the initial crosses to allow for the emergence of new allelic combinations in the bean line selection program. The data generated from the molecular analysis proved to be effective, providing a real scenario of genetic diversity contained in the gene pool integrated by lines used in the VCU tests of the common bean. This result allows for new strategies to be planned to increase the genetic base from which the program is conducted and, therefore, to increase the genetic gain per selection cycle to minimize the risk of vulnerability to biotic and abiotic stresses and the occurrence of low levels of productivity.

\section{ACKNOWLEDGMENTS}

Research supported by Conselho Nacional de Desenvolvimento Científico e Tecnológico (CNPq; grants awarded to C. Brondani, P.C.B. Cardoso, L.C. Melo and R.P. Vianello) and EMBRAPA/MP2. We thank Gesimaria Ribeiro Costa Coelho for the technical assistance with the ABI3100 platform (Applied Biosystems) at Embrapa Arroz e Feijão, Dr. Helton Santos Pereira and João Antônio Mendonça for the common bean seed supply.

\section{REFERENCES}

Abreu AFB, Ramalho MAP, Santos JB and Martins LA (2004). Progresso do melhoramento genético do feijoeiro nas décadas de setenta e oitenta nas regiões Sul e Alto Paranaíba em Minas Gerais. Pesq. Agropec. Bras. 29: 105-112.

Belkhir K, Borsa P, Chikhi L, Raufaste N, et al. (2004). GENETIX 4.05, logiciel sous Windows TM pour la génétique des populations. Version 4.05. Laboratoire Génome, Populations, Interactions, CNRS UMR 5171. Université de Montpellier II, Montpellier.

Benchimol LL, Campos T, Carbonell SAM, Colombo CA, et al. (2007). Structure of genetic diversity among common bean (Phaseolus vulgaris L.) varieties of Mesoamerican and Andean origins using new developed microsatellite markers. Genet. Resour. Crop Evol. 54: 1747-1762.

Blair MW, Torres MM, Giraldo MC and Pedraza F (2009). Development and diversity of Andean-derived, gene-based microsatellites for common bean (Phaseolus vulgaris L.). BMC Plant Biol. 9: 100.

Blair MW, Hurtado N, Chavarro CM, Munoz-Torres MC, et al. (2011). Gene-based SSR markers for common bean (Phaseolus vulgaris L.) derived from root and leaf tissue ESTs: an integration of the BMc series. BMC Plant Biol. 11: 50.

Borém A and Carneiro JES (2006). A cultura. In: Feijão (Vieira C, Paula TJ Jr and Borém A, eds.). 2nd edn. Atual, Viçosa, 13-18.

Broughton WJ, Hernández G, Blair M, Beeb S, et al. (2003). Beans (Phaseolus spp.) - model food legumes. Plant Soil 252: $55-128$.

Bueno LG, Pereira HS, Melo LC, Wendland A, et al. (2011). Genotypes of common beans export type evaluated in Brazil. Annu. Rep. Bean Improv. Coop. 54: 62-63.

Buso GSC, Amaral ZPS, Brondani RPV and Ferreira ME (2006). Microsatellite markers for the common bean Phaseolus vulgaris. Mol. Ecol. Notes 6: 252-254.

Butler JM (2006). Genetics and genomics of core short tandem repeat loci used in human identity testing. J. Forensic Sci. 51: 253-265.

Cardoso PCB (2008). Desenvolvimento de um Sistema de Genotipagem Multiplex Baseado em Marcadores Microssatélites para a Caracterização Molecular de Cultivares e de Linhagens de Ensaios de Valor de Cultivo e Uso (VCU) de Feijoeiro Comum (Phaseolus vulgaris). Master's thesis, Universidade Federal de Goiás, Goiânia.

Chiorato AF (2008). Progresso Genético para Produtividade do Feijoeiro no Programa de Melhoramento do Instituto Agronômico (IAC) entre 1989 e 2007. Doctoral thesis, Escola Superior de Agricultura "Luiz de Queiroz", Universidade de São Paulo, Piracicaba. 
CONAB (Companhia Nacional de Abastecimento) (2008). Available at [http://www.conab.gov.br/conabweb] Accessed January 7, 2012.

Earl DA and VonHold BM (2011). Structure Harvester: a website and program for visualizing STRUCTURE output and implementing the Evanno method. Conserv. Genet. Resour. 4: 359-361.

Evanno G, Regnaut S and Goudet J (2005). Detecting the number of clusters of individuals using the software STRUCTURE: a simulation study. Mol. Ecol. 14: 2611-2620.

Falush D, Stephens M and Pritchard JK (2003). Inference of population structure using multilocus genotype data: linked loci and correlated allele frequencies. Genetics 164: 1567-1587.

FAO (Food and Agriculture Organization of the United Nations) (2008). Statistical Database. Available at [http://www. fao.org/faostat] Accessed January 10, 2011.

Faria JC, Carneiro GE and Aragao FJ (2010). Gene flow from transgenic common beans expressing the bar gene. GM Crops 1: 94-98.

Ferreira CM, Santos ML, Braga MJ and Del Peloso MJ (2006). Aspectos Econômicos. In: Feijão (Vieira C, Paula TJ Jr and Borém A, eds.). 2nd edn. Ed. UFV, Viçosa, 19-40.

Ferreira JL, Carneiro JES, Teixeira AL, Lanes FF, et al. (2007). Gene flow in common bean (Phaseolus vulgaris L.). Euphytica 153: 165-170.

Ferreira ME and Grattapaglia D (1998). Introdução ao Uso de Marcadores Moleculares em Análise Genética. Embrapa, Brasília.

Gaitán-Solís E, Duque MC, Edwards KJ and Tohme J (2002). Microsatellite repeats in common bean (Phaseolus vulgaris): Isolation, characterization and cross-species amplification in Phaseolus ssp. Crop Sci. 42: 1228-1236.

Garcia RA, Rangel PN, Brondani C, Martins WS, et al. (2011). The characterization of a new set of EST-derived simple sequence repeat (SSR) markers as a resource for the genetic analysis of Phaseolus vulgaris. BMC Genet. 12: 41.

Goudet J (2002). FSTAT: a program to estimate and test gene diversities and fixation indices (version 2932). Avaliable at [http://www2.unil.ch/popgen/softwares/fstat.htm]. Accessed July 10, 2011.

Grisi MC, Blair MW, Gepts P, Brondani C, et al. (2007). Genetic mapping of a new set of microsatellite markers in a reference common bean (Phaseolus vulgaris) population BAT93 x Jalo EEP558. Genet. Mol. Res. 6: 691-706.

Gupta PK, Balyan HS, Sharma PC and Ramesh B (1996). Microsatellites in plants: A new class of molecular markers. Curr. Sci. 70: 45-54.

Lewis PO and Zaykin D (2001). Genetic Data Analysis: Computer program for the analysis of allelic data. (Version 1.0). Available at [http://lewis.eeb.uconn.edu/lewishome/software.html/]. Accessed December 15, 2011.

MAPA (Ministério da Agricultura, Pecuária e Abastecimento) (2010). Informações aos Usuários de Proteção de Cultivares. Available at [http://www.agricultura.gov.br/arq_editor/file/INFORMACOES_AOS_USUARIOS_SNPC_nov2010. pdf] Accessed January 20, 2013.

MAPA (Ministério da Agricultura Pecuária e Abastecimento) (2011). Brasil Projeções do Agronegócio 2010/2011 a 2020/2021. Available at [http://www.agricultura.gov.br/arq_editor/file/Ministerio/gestao/projecao/PROJECOES\%20 DO\%20AGRONEGOCIO\%202010-11\%20a\%202020-21\%20-\%202_0.pdf]. Accessed November 20, 2011.

Marinho CD, Martins FJ, Amaral SC, Amaral Junior AT, et al. (2011). Revisiting the Brazilian scenario of registry and protection of cultivars: an analysis of the period from 1998 to 2010, its dynamics and legal observations. Genet. Mol. Res. 10: 792-809.

Masi P, Spagnoletti Zeuli PL and Donini P (2003). Development and analysis of multiplex microsatellite markers sets in common bean (Phaseolus vulgaris L.). Mol. Breed. 11: 303-313.

Matos JW (2005). Análise Crítica do Programa de Melhoramento Genético do Feijoeiro da UFLA no Período de 1974 a 2004. Doctoral thesis. UFLA, Lavras.

Métais I, Hamon B, Jalouzot R and Peltier D (2002). Structure and level of genetic diversity in various bean types evidenced with microsatellite markers isolated from a genomic enriched library. Theor. Appl. Genet. 104: 1346-1352.

Nei M (1987). Molecular Evolutionary Genetics. 1st edn. Columbia University Press, New York.

Pessoa-Filho M, Belo A, Alcochete AA, Rangel PH, et al. (2007). A set of multiplex panels of microsatellite markers for rapid molecular characterization of rice accessions. BMC Plant Biol. 7: 23.

Pritchard JK, Stephens M and Donnelly P (2000). Inference of population structure using multilocus genotype data. Genetics 155: 945-959.

Ramalho MAP, Santos JB and Pinto CABP (2004). Genética na Agropecuária. Universidade de Lavras, Lavras.

Rangel PHN, Guimarães EP and Neves PCF (1996). Base genética das cultivares de arroz (Oryza sativa L.) irrigado do Brasil. Pesq. Agropec. Bras. 31: 349-357.

Rohlf FJ (1993). NTSYS-pc, numerical taxonomy and multivariate analysis system. Version 2.02g. Exeter Software, Setauket.

Sayama T, Hwang TY, Komatsu K, Takada Y, et al. (2011). Development and application of a whole-genome simple sequence repeat panel for high-throughput genotyping in soybean. DNA Res. 18: 107-115. 
Wagner HW and Sefc KM (1999). Identity, 1.0. Wienn: Centre for Applied Genetics. University of Agricultural Sciences, Vienna.

Zizumbo-Villarreal D, Colunga-GarcíaMarín P, Payró de la Cruz E, Delgado-Valerio P, et al. (2005). Population structure and evolutionary dynamics of wild - weedy - domesticated complexes of common bean in a Mesoamerican Region. Crop Sci. 45: 1073-1083. 